\title{
Half-Gate Light-Emitting Electrochemical Transistor to Achieve Centered Emissive Organic p-n Junction
}

Jiang Liu, Isak Engquist and Magnus Berggren

\author{
Linköping University Post Print
}

\section{Tweet}

N.B.: When citing this work, cite the original article.

Original Publication:

Jiang Liu, Isak Engquist and Magnus Berggren, Half-Gate Light-Emitting Electrochemical Transistor to Achieve Centered Emissive Organic p-n Junction, 2014, Organic electronics, 18, pp 32-36.

http://dx.doi.org/10.1016/j.orgel.2014.12.027

Copyright: Elsevier

http://www.elsevier.com/

Postprint available at: Linköping University Electronic Press http://urn.kb.se/resolve?urn=urn:nbn:se:liu:diva-104924 


\title{
Half-Gate Light-Emitting Electrochemical Transistor to Achieve Centered Emissive Organic p-n Junction
}

Jiang Liu, Isak Engquist and Magnus Berggren

Laboratory of Organic Electronics, Department of Science and Technology, Linköping University, SE-601 74 Norrköping

\begin{abstract}
Organic light-emitting electrochemical cells (LEC) are promising for lighting applications but in many cases these devices suffer from unbalanced electrochemical doping, which cause instability and degradation of the cells. A predominant p-doping over n-doping causes an off-centered emissive $p$-n junction, which leads to poor powerconversion efficiency. Here, we report a half-gate LEC transistor (HG-LECT), in which an ion-conductive gate made from poly(3,4-ethylenedioxythiophene)-poly(styrenesulfonate) is employed to combat this instability problem. The gate material, covering half of the channel, is used to enhance the n-doping process in this part by employing an appropriate operation protocol. We demonstrate a centered light emission zone, closely following the geometry of the gate configuration. The HG-LECT with centered emission profile is shown to be more efficient than the corresponding LEC without the gate electrode, and its n-doping level is measured to be $15 \%$.
\end{abstract}

Keywords: Light-emitting electrochemical transistor; light-emitting electrochemical cell; PEDOT:PSS; MEH-PPV; polymer electrolyte.

\section{Introduction}

The electrical conductivity and electroluminescence of organic conjugated semiconductors, together with their processability, such as using inexpensive solution-based fabrication, are together attractive properties that may pave the way for radically novel light-sources. After the invention of organic light-emitting diodes in $1990^{1}$, other organic optoelectronic devices have been demonstrated, such as lightemitting electrochemical cells (LEC) ${ }^{2-4}$ and light-emitting transistors (LET) ${ }^{5-8}$.

Conventional LECs include a single electroluminescent layer sandwiched between two charge-injection electrodes. The electroluminescent material is usually a mixture of a light emitting polymer (LEP) and an electrolyte. Upon application of a voltage difference at the electrodes, ions redistribute within the LEP, leading to a p-doped region close to the anode and an n-doped region close to the cathode. The injected electrons and holes flow through the $\mathrm{n}$ - and $\mathrm{p}$-doped LEP and recombine at the organic $\mathrm{p}$-n junction to generate light emission.

Several groups have tried to apply the transistor structure in LECs, in order to simultaneously modulate the doping profile in the LEP material, the charge transport 
characteristics and photon generation. Adding a metal gate and a conventional gate insulator (such as inorganic $\mathrm{SiO}_{2}$ or organic materials poly(vinyl alcohol) ) to the LEC structure enables the control of electric current ${ }^{9-10}$ as well as the intensity of the luminescence ${ }^{11}$. However, this approach requires a high operating voltage at the gate electrode as the electrostatic coupling between the gate and the LEP is weak. Bhat et al. employed a metal gate with an ion gel as gate insulator on the foundation of an $\mathrm{LEP}^{12}$. This transistor configuration leads to low gate-voltage operation due to the electrochemical doping between gate and LEP, but the device displays an emissive zone in proximity to the electron injection electrode (cathode), showing a lack of ndoping in the LEP. Indeed, a light-emitting junction adjunct to the cathode is one of the common drawbacks in LECs ${ }^{13-16}$, as it leads to photon quenching at the electrode and decreases the power-conversion efficiency of luminescence in the device. The lack of $n$-doping is mainly due to that the reactants, which are involved in the n-doping process, are partially consumed in undesired electrochemical or chemical sidereactions ${ }^{15,17}$ resulting in a dominant $\mathrm{p}$-doping. To achieve balanced $\mathrm{p}$ - and $\mathrm{n}$-doping for a centered emissive junction, n-doping needs to be favored. We have previously developed a class of light-emitting electrochemical transistors (LECT), with a polymer electrolyte as the gate insulator and a poly(3,4-ethylenedioxythiophene)-poly(styrenesulfonate) (PEDOT:PSS) as the gate electrode. The first generation of those LECTs includes a PEDOT:PSS gate that fully covers the channel. It was shown that both the $\mathrm{p}$ - and the $\mathrm{n}$-doping region can be modified by the gate electrode, and an emissive $p-n$ junction can be spatially controlled within the LEP channel ${ }^{18}$. The second generation of LECT features two gates, which controls n- and p-doping separately; it demonstrates a more static and centered p-n junction located between the two gates ${ }^{19}$, thanks to a more balanced doping profile. The double-gate LECT provides a platform to study the physics of LECs when n-doping and p-doping are decoupled. However, if one only desires a centered emission zone aiming to maximize the efficiency in practical applications and to stabilize the emitting zone, we found that in the double gate configuration, the gate electrode responsible for controlling the p-doping can in fact be omitted. In this letter, we consequently demonstrate a half-gate LECT (HGLECT), with a gate electrode partially covering the LEP channel that controls only the n-doping process. In this device, we demonstrate that a centered emission zone is achieved after a two-step operation protocol. We also compare the efficiency of devices with and without the gate electrode. 


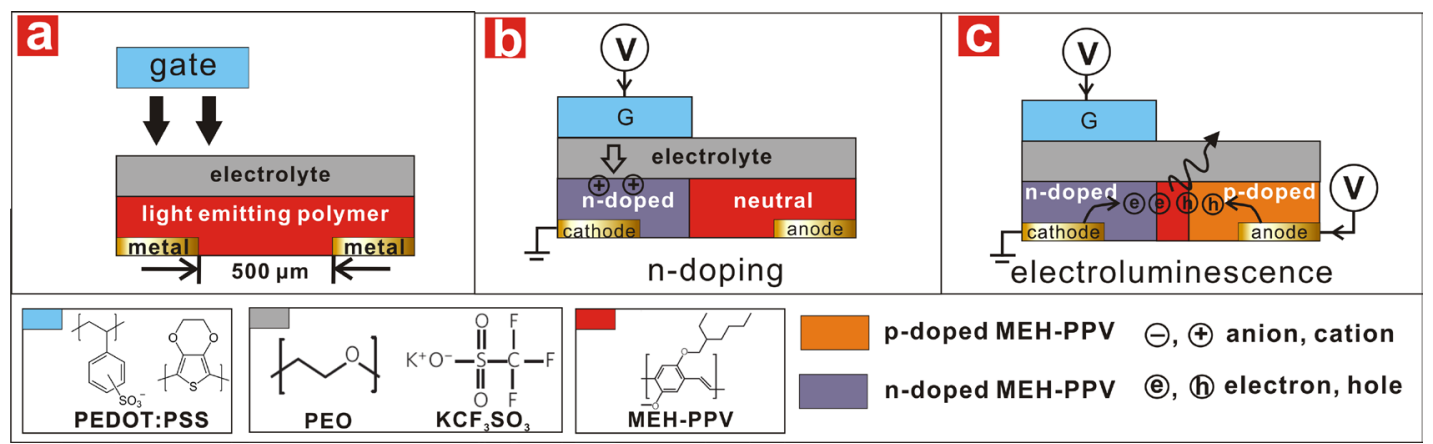

Figure 1. The structure and operation modes of HG-LECT.

\section{Structure, Materials and Operation Modes}

The HG-LECT, as shown in Figure 1a, was fabricated on a $\mathrm{SiO}_{2} / \mathrm{Si}$ substrate, where $\mathrm{Au}$ electrodes with a spacing of $500 \mu \mathrm{m}$ were thermally evaporated through a shadow mask. Poly[2-methoxy-5-(2-ethylhexyloxy)-1,4-phenylenevinylene] (MEH-PPV, Mn=40,000-70,000, Sigma-Aldrich) dissolved in chloroform with a concentration of $10 \mathrm{mg} / \mathrm{ml}$ was spin-coated onto the substrate at $1000 \mathrm{rpm}$ for $60 \mathrm{~s}$, and then dried at $90{ }^{\circ} \mathrm{C}$ for $100 \mathrm{~s}$, forming a dry film of $200 \mathrm{~nm}$ thickness. A drop of the electrolyte solution was then employed onto the MEH-PPV film, before a freestanding sheet of PEDOT:PSS (Orgacon EL-350, Agfa) was pressed onto the electrolyte drop with the PEDOT:PSS electrode facing down. The electrolyte solution was made by blending poly(ethylene oxide) (PEO, $\mathrm{Mw}=600,000$, Aldrich) and $\mathrm{CF}_{3} \mathrm{SO}_{3} \mathrm{~K}$ (98\%, Aldrich) with a mass ratio 5.4:1 into a $3: 1 \mathrm{v} / \mathrm{v}$ mixture of 1-propanol (Sigma-Aldrich) and deionized water, followed by stirring on a hotplate at $90{ }^{\circ} \mathrm{C}$ for $24 \mathrm{~h}$. Finally, the device was dried inside a cryostat, under a vacuum of $2 \times 10^{-5}$ mbar at $70^{\circ} \mathrm{C}$, for 12 hours. The bottom electrode directly beneath the gate electrode is here termed the cathode, and the other bottom electrode is termed the anode.

Two operation modes of the HG-LECT are introduced as follows: $n$-doping mode and electroluminescence(EL) mode. In $\mathrm{n}$-doping mode, a voltage of $4 \mathrm{~V}$ is applied at the gate electrode in reference to the cathode, with the anode kept floating; see Figure 1b. The further oxidation of PEDOT, in the PEDOT:PSS gate electrode, causes a cation migration within the electrolyte layer, which diffuses into the LEP layer causing reduction the conjugated polymer. By applying this process for sufficient amount of time, the part of the LEP below the gate terminal becomes $n$-doped, leaving the part of the LEP without gate coverage neutral. In EL mode(followed by n-doping mode), a potential of $4 \mathrm{~V}$ is applied between the anode and the cathode, with the gate voltage of $4 \mathrm{~V}$ still applied. This operation promotes $\mathrm{p}$-doping of the previously neutral part of the LEP and also further enhances the n-doping level of LEP material located under the gate. Since the n-doping progresses relatively slower than $\mathrm{p}$-doping, we expect that the p-n junction forms in close vicinity of the edge of the gate terminal, as shown in Figure 1c. The application of the gate voltage continuously enhances the n-doping territory so that the $\mathrm{p}-\mathrm{n}$ junction is stable after it is formed. 
Electrical characterization of the HG-LECT was performed under vacuum in a cryostat using a Keithley 4200 Semiconductor Characterization System. An HGLECT with the gate covering 50\% of the channel (as shown in Figure 2a) was operated following a two-step protocol: (1) $100 \mathrm{~s}$ of n-doping mode, by application of $4 \mathrm{~V}$ at the gate terminal and (2) $1000 \mathrm{~s}$ of EL mode by application of $4 \mathrm{~V}$ at the gate and anode terminals. The time for $\mathrm{n}$-doping was chosen so that the $\mathrm{n}$-doping level would reach the same level as in a conventional LEC (details of the calculation in the discussion section). We chose to operate the device at $4 \mathrm{~V}$ in order to overcome the bandgap of MEH-PPV ( 2.3 eV) and promote efficient charge injection. The temperature during the measurement was kept at $70^{\circ} \mathrm{C}$, which is above the glass transition temperature of the ion conductor $\mathrm{PEO}\left(65^{\circ} \mathrm{C}\right)$, so that the ions are mobile at this temperature.

a

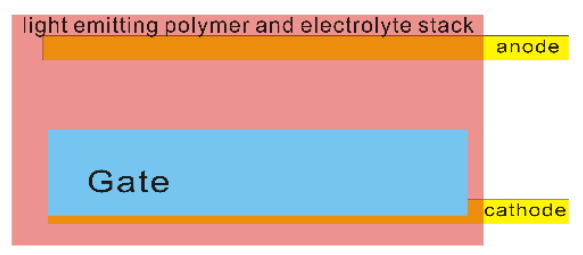

b

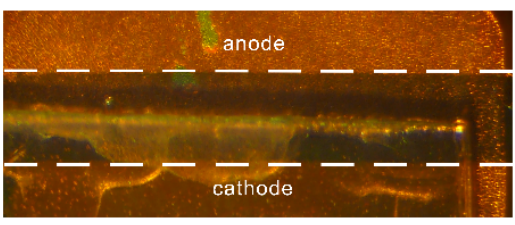

C

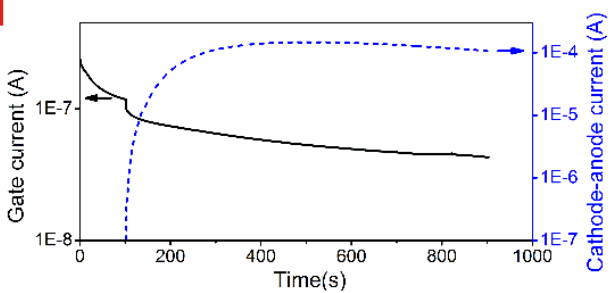

(c)

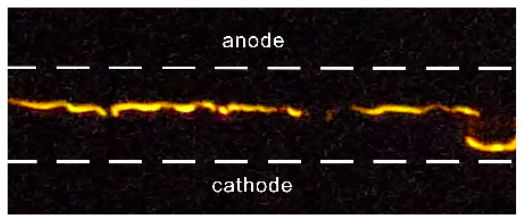

Figure 2. (a) Sketch and (b) photo of the HG-LECT in top view; (c) the temporal change of current under the two operation modes; (d) photo of the light emission zone in EL mode.

\section{Experimental Results}

The electrical measurement data is displayed in Figure 2c. The solid line indicates the gate current during both the operational modes, while the dashed curve shows the cathode-anode current during EL mode operation. A sudden decrease of the gate current can also be observed at $100 \mathrm{~s}$, indicating a clear influence of the application of the cathode voltage. Light emission occurs approximately 3 seconds after the EL mode operation is initiated. As shown in Figure 2d, the emissive junction aligns well with the edge of the gate, which proves that the $p-n$ junction is established at that position. During the following $1000 \mathrm{~s}$ of EL mode operation, the emissive junction remains at the same position. For the purpose of comparison, a small portion of the channel is 
left uncovered by the gate electrode. This part of the channel displays an emissive junction close to the cathode, as displayed in the right part of Figure $2 \mathrm{~d}$.
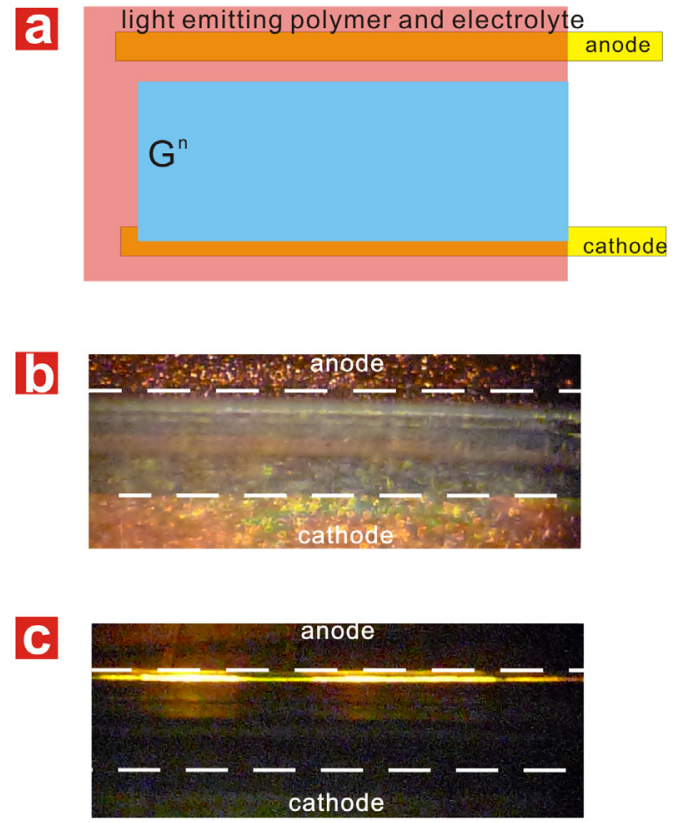

Figure 3. (a) Sketch and the (b) photo of an HG-LECT, in which the gate covers $90 \%$ of the channel. (c) The emissive zone during EL mode operation.

Additionally, an HG-LECT was made with the gate electrode covering about $90 \%$ of the channel, as shown in the sketch and in the photo given in Figure $3 \mathrm{a}$ and $3 \mathrm{~b}$. Using a similar operation procedure as with the previous device, a highly uniform emissive zone was established close to the anode, again well aligned with the edge of the gate electrode, as shown in Figure 3c. This further proves that the HG-LECT enables a light emission zone according to the geometry and position of the gate electrode.

One of the purposes of the Hg-LECT is to reduce the quenching of photons at the electrode in order to achieve high power-conversion efficiency. To test this hypothesis, we compare two devices during EL mode operation: one HG-LECT device with 50\% gate coverage and one LEC device ${ }^{20}$ with planar cathode-anode electrodes (i.e. an HGLECT without gate electrode). The HG-LECT is n-doped by application of $4 \mathrm{~V}$ to the gate using the cathode as reference, for $100 \mathrm{~s}$, prior to the EL mode operation. In the EL mode, a constant current of $1 \mu \mathrm{A}$ is applied between the anode and the cathode. The same current is also applied to the LEC device. A photodiode was used to collect the photocurrent, see Figure 4. The LEC device shows relatively higher light emission efficiency at the beginning of the application of the voltage but then drops gradually. The HG-LECT shows a lower efficiency during the first $100 \mathrm{~s}$, but then displays an increasing trend and becomes more efficient than the LEC after $100 \mathrm{~s}$. The integration 
of current over time indicates the average efficiency of the HG-LECT exceeds that of the LEC by $40 \%$.

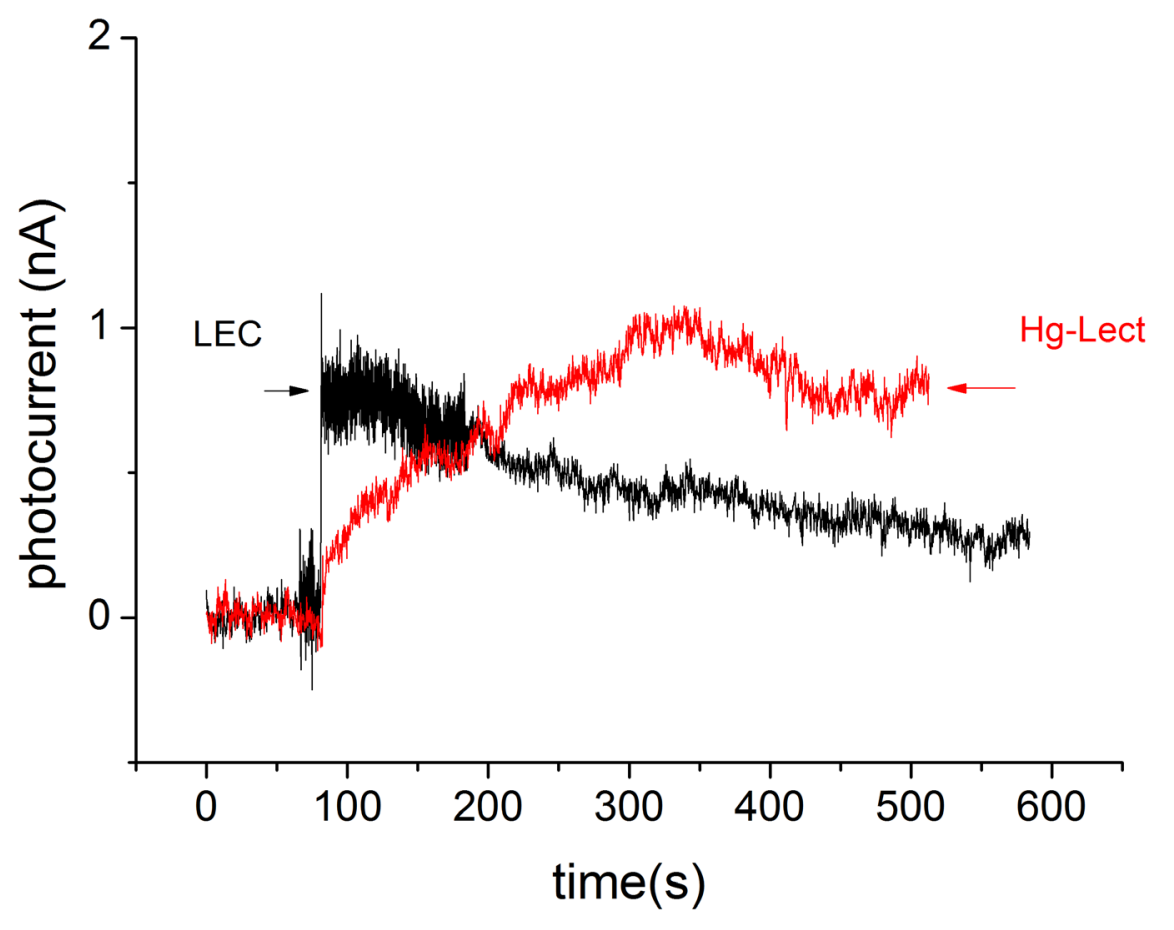

Figure 4. The photo current measurement for an LEC and an HG-LECT during EL mode operation with identical current applied. The HG-LECT was operated in ndoping mode prior to the EL mode.

\section{Discussion}

It is important to achieve a sufficient doping level in the LEP during n-doping mode before operating the device in EL mode. The doping level is evaluated as follows:

We define the doping level as the ratio of doped ppv units to all ppv units:

$$
\text { Doping level }=\frac{n_{\text {doped }-p p v}}{n_{p p v}}
$$

To estimate the amount of doped ppv, we assume that in n-doping mode, all the current flowing through the gate to the cathode is involved in electrochemical doping of PPV, and each charge carrier that is injected from the gate electrode results in one cation that migrates from the electrolyte layer to the LEP, leading to one n-doped PPV repeat unit. We then obtain $n_{\text {doped-ppv }}$ from the following formula:

$$
n_{\text {doped-ppv }}=\frac{Q_{\text {doping }}}{e}=\frac{\int I_{\text {doping }}}{e}
$$


The total number of $\mathrm{ppv}$ repeat units in the $\mathrm{n}$-doped area $\mathrm{n}_{\mathrm{ppv}}$ can be calculated by

$$
n_{p p v}=\frac{m_{p p v}}{M_{p p v}} \times N_{A}
$$

Where $\mathrm{N}_{\mathrm{A}}$ is Avogadro's constant $\left(6.22^{*} 10^{-23} \mathrm{~mol}^{-1}\right), \mathrm{m}_{\mathrm{ppv}}$ is the mass of ppv $\left(3^{*} 10^{-7} \mathrm{~g}\right)$ in the $n$-doped portion of the device (beneath the gate electrode) and $M_{p p v}$ is the molar mass of the repeat unit $(261 \mathrm{~g} / \mathrm{mol})$.

Finally the doping level can be calculated by the following:

$$
\text { Doping level }=\frac{n_{\text {doped }-p p v}}{n_{p p v}}=\frac{\frac{\int I_{\text {doping }}}{e}}{\frac{m_{p p v}}{M_{p p v}} \times N_{A}}=\frac{\int I_{\text {doping }}}{1.144 * 10^{-4}}
$$

Using this method, we obtain an n-doping level of the HG-LECT device discussed in Figure 2 of $15 \%$, which is comparable to a conventional LEC as reported by Edman and coworkers ${ }^{15,21 .}$

By evaluating the doping level in different experiments, we confirm empirically that a doping level of 13\%-15\% suffices for the device to achieve a centered emission zone. A doping level lower than $13 \%$ results in a non-homogenous emissive zone, which typically is manifested as a non-straight line underneath the gate electrode and close to the cathode, see Figure S1-b in Supporting Information.

\section{Conclusion}

In summary, we demonstrate a half-gate LECT device, in which the emissive junction formation can be controlled by employing a two-step operational scheme, and where the shape of the gate electrode controls the position of said emission zone. Furthermore, we show that an HG-LECT with a centered emission zone outperforms the conventional bilayer LEC in terms of power-conversion efficiency. We also obtain an approximate $\mathrm{n}$-doping level of $\sim 15 \%$ during $\mathrm{n}$-doping mode operation of the HGLECT.

\section{Acknowledgment}

This work has been supported by the Swedish Foundation for Strategic Research (OPEN), the European Regional Development Fund through Tillväxtverket (PEAPPP), VINNOVA 2012-01607 (PEA), and Knut and Alice Wallenberg Foundation (Power Papers). M.B. acknowledges the Önnesjö Foundation for financial support. J.L. is thankful to Robert Forchheimer for valuable discussions. 


\section{References}

1. Burroughes, J.; Bradley, D.; Brown, A.; Marks, R.; Mackay, K.; Friend, R.; Burns, P.; Holmes, A., Light-Emitting Diodes Based on Conjugated Polymers. nature 1990, 347, 539-541.

2. Pei, Q.; Yu, G.; Zhang, C.; Yang, Y.; Heeger, A. J., Polymer Light-Emitting Electrochemical Cells. Science 1995, 269, 1086-1088.

3. Shoji, T. D.; Zhu, Z.; Leger, J. M., Characterizing Ion Profiles in Dynamic Junction LightEmitting Electrochemical Cells. ACS Appl Mater Interfaces 2013, 5, 11509-14.

4. Wantz, G.; Gautier, B.; Dumur, F.; Phan, T. N. T.; Gigmes, D.; Hirsch, L.; Gao, J., Towards Frozen Organic Pn Junctions at Room Temperature Using High-Tg Polymeric Electrolytes. Org. Electron. 2012, 13, 1859-1864.

5. Zaumseil, J.; Friend, R. H.; Sirringhaus, H., Spatial Control of the Recombination Zone in an Ambipolar Light-Emitting Organic Transistor. Nat. Mater. 2006, 5, 69-74.

6. Seo, J. H.; Namdas, E. B.; Gutacker, A.; Heeger, A. J.; Bazan, G. C., Solution-Processed Organic Light-Emitting Transistors Incorporating Conjugated Polyelectrolytes. Adv. Funct. Mater. 2011, 21, 3667-3672.

7. Hsu, B. B.; Duan, C.; Namdas, E. B.; Gutacker, A.; Yuen, J. D.; Huang, F.; Cao, Y.; Bazan, G. C.; Samuel, I. D.; Heeger, A. J., Control of Efficiency, Brightness, and Recombination Zone in Light-Emitting Field Effect Transistors. Adv. Mater. 2012, 24, 1171-5.

8. Cicoira, F.; Santato, C., Organic Light Emitting Field Effect Transistors: Advances and Perspectives. Adv. Funct. Mater. 2007, 17, 3421-3434.

9. Yumusak, C.; Sariciftci, N. S., Organic Electrochemical Light Emitting Field Effect Transistors. Appl. Phys. Lett. 2010, 97, 033302-3.

10. Edman, L.; Swensen, J.; Moses, D.; Heeger, A. J., Toward Improved and Tunable Polymer Field-Effect Transistors. Appl. Phys. Lett. 2004, 84, 3744.

11. Yumusak, C.; Abbas, M.; Sariciftci, N. S., Optical and Electrical Properties of Electrochemically Doped Organic Field Effect Transistors. J Lumin 2013, 134, 107-112.

12. Bhat, S. N.; Di Pietro, R.; Sirringhaus, H., Electroluminescence in Ion-Gel Gated Conjugated Polymer Field-Effect Transistors. Chem. Mater. 2012, 24, 4060-4067.

13. Matyba, P.; Maturova, K.; Kemerink, M.; Robinson, N. D.; Edman, L., The Dynamic Organic P-N Junction. Nat. Mater. 2009, 8, 672-676.

14. Sakanoue, T.; Sawabe, K.; Yomogida, Y.; Takenobu, T.; Seki, S.; Ono, S., Optically Pumped Amplified Spontaneous Emission in an Ionic Liquid-Based Polymer Light-Emitting Electrochemical Cell. Appl. Phys. Lett. 2012, 100, 263301.

15. Fang, J.; Matyba, P.; Robinson, N. D.; Edman, L., Identifying and Alleviating Electrochemical Side-Reactions in Light-Emitting Electrochemical Cells. J. Am. Chem. Soc. 2008, 130, 4562-4568.

16. Rodovsky, D. B.; Reid, O. G.; Pingree, L. S. C.; Ginger, D. S., Concerted Emission and Local Potentiometry of Light-Emitting Electrochemical Cells. ACS Nano 2010, 4, 2673-2680.

17. Fang, J.; Yang, Y.; Edman, L., Understanding the Operation of Light-Emitting Electrochemical Cells. Appl. Phys. Lett. 2008, 93.

18. Liu, J.; Engquist, I.; Crispin, X.; Berggren, M., Spatial Control of P-N Junction in an Organic Light-Emitting Electrochemical Transistor. J. Am. Chem. Soc. 2011, 134, 901-904.

19. Liu, J.; Engquist, I.; Berggren, M., Double-Gate Light-Emitting Electrochemical Transistor: Confining the Organic P-N Junction. J. Am. Chem. Soc. 2013, 135, 12224-12227.

20. Sandström, A.; Matyba, P.; Inganäs, O.; Edman, L., Separating Ion and Electron Transport: The Bilayer Light-Emitting Electrochemical Cell. J. Am. Chem. Soc. 2010, 132, 6646-6647. 
21. Shin, J. H.; Robinson, N. D.; Xiao, S.; Edman, L., Polymer Light-Emitting Electrochemical Cells: Doping Concentration, Emission-Zone Position, and Turn-on Time. Adv. Funct. Mater. 2007, 17, 1807-1813. 


\title{
Supporting Information
}

\section{Half-Gate Light-Emitting Electrochemical Transistor to Achieve Centered Emissive Organic p-n Junction}

\author{
Jiang Liu, Isak Engquist and Magnus Berggren*
}

Laboratory of Organic Electronics, Department of Science and Technology, Linköping University, SE-601 74 Norrköping, Sweden

Email: Magnus.Berggren@liu.se

\section{EL mode of HG-LECT after different amounts of $n$-doping.}

Two HG-LECTs with identical geometries as shown in Figure S1-a were operated in the sequence of $n$-doping and EL mode. In the first HG-LECT, $100 \mathrm{~s}$ of $\mathrm{n}$-doping results in a centered and uniform light emission in EL mode (shown in Figure $2 d$ in the article and redrawn in Figure S1-b for comparison), with a doping level of $15 \%$ in n-doping mode. In the second device, a shorter duration (70 s) of n-doping was applied before EL mode, which leads to a non-centered and inhomogeneous emissive profile (Figure S1-d). The electrical data of the second device is shown in figure S1-c, from which the doping level of the n-doping mode was calculated to be $12 \%$, using the method mentioned in the Discussion part of the manuscript.
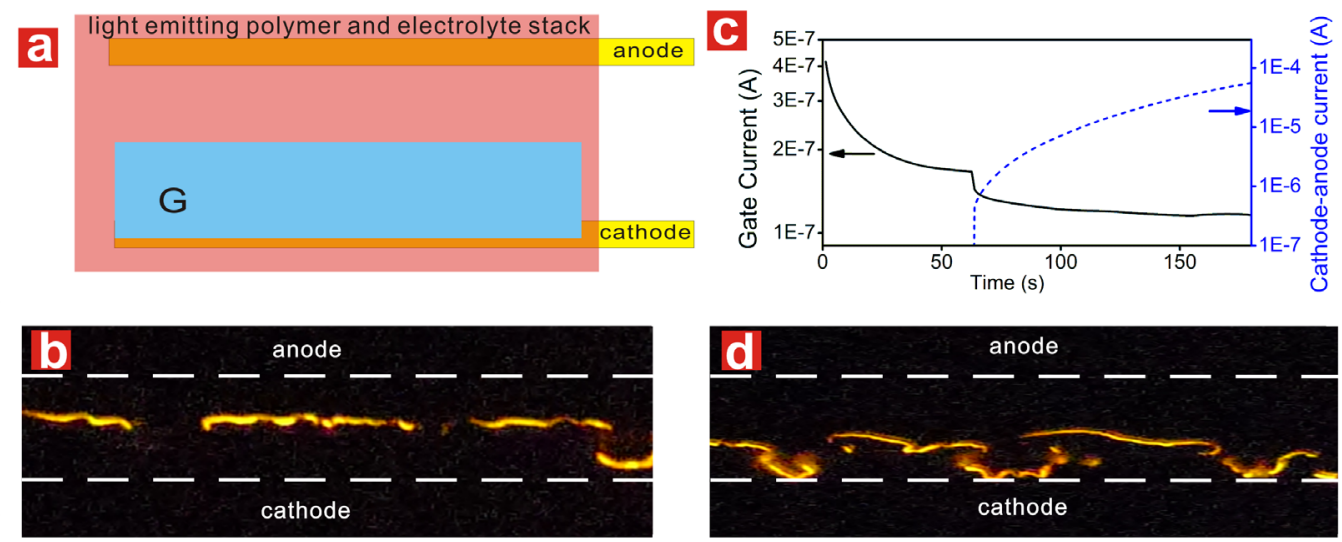

Figure S1. (a). Sketch of HG-LECTs with gate covering $50 \%$ of the LEP. Light emission under (b) sufficient and (d) insufficient $n$-doping. The electrical characterization of the insufficiently doped sample is shown in (c). 